\title{
Effect of calving process on the outcomes of delivery and postpartum health of dairy cows with unassisted and assisted calvings
}

\author{
L. Kovács, ${ }^{*} \dagger^{1}$ F. L. Kézér, ${ }^{*} \dagger$ and O. Szenci†¥ \\ *Magyar Tudományos Akadémia-Szent István Egyetem, Large Animal Clinical Research Group, Üllö-Dóra major 2225, Hungary \\ †Institute of Animal Husbandry, Faculty of Agricultural and Environmental Science, Szent István University, Páter Károly utca 1, Gödöllö 2100, \\ Hungary \\ ‡Department and Clinic for Production Animals, Faculty of Veterinary Science, Szent István University, Üllö-Dóra major, H-2225, Hungary
}

\begin{abstract}
Welfare aspects of obstetrical assistance were studied in multiparous Holstein-Friesian cows $(\mathrm{n}=176)$ with (1) unassisted calving in an individual pen (UCIP; n $=42$ ), (2) unassisted calving in a group pen (UCG; $\mathrm{n}$ $=48)$, (3) assisted calving with appropriately timed obstetrical assistance (ACAP; $\mathrm{n}=50$ ), and (4) assisted calving with inappropriately timed (premature) obstetrical assistance (ACIN; $\mathrm{n}=36$ ). Duration of the stages of calving, the prevalence and the degree of dystocia, stillbirth ratio, newborn calf vitality, and the occurrence of postpartum health problems (i.e., retained placenta and vulvovaginal laceration) were recorded. The time from amniotic sac and hooves appearance to birth and the total duration of calving (from the onset of calving restlessness to delivery) were shorter for UCG cows than for any other groups. The overall incidence of dystocia was $31.3 \%$ in the calvings studied. The prevalence of dystocia was below $10 \%$ in cases of unassisted calvings. The proportion of severe dystocia was higher in ACIN cows than in ACAP cows (47.2 vs. $12.0 \%$, respectively). The prevalence of stillbirths was the highest in ACIN calvings $(22.2 \%)$, followed by ACAP, UCI, and UCG cows $(8.0,4.8$, and $0.0 \%$, respectively). The ACIN calves had lower vitality scores than calves born from ACAP, UCG, and UCIP dams immediately after delivery and $24 \mathrm{~h}$ after birth. Although ACAP calves had lower vitality scores than UCG and UCIP calves at birth, a delayed recovery of vitality was mirrored by satisfactory vitality scores $24 \mathrm{~h}$ after birth. Retained placenta and vulvovaginal laceration occurred more often with assisted dams (i.e., ACAP and ACIN animals) compared with UCIP cows with the highest prevalence in ACIN cows. In UCG cows, no injuries occurred in the vulva or vagina, and we noted only
\end{abstract}

Received April 16, 2016.

Accepted June 2, 2016.

${ }^{1}$ Corresponding author: Kovacs.Levente@mkk.szie.hu
4 cases of retained placenta $(8.3 \%)$, proportions lower than in cows with unassisted calving in the maternity pen. Our results suggest that calving in a group might have benefits over calving in an individual pen in terms of calving ease, duration of the delivery process, and postpartum health of the dam vitality of her offspring. Premature obstetrical assistance leads to a high prevalence of dystocia, impairs postpartum health of the dam, and poses a potential risk to calf survival.

Key words: calving, obstetrical assistance, postpartum health, dystocia, newborn calf vitality

\section{INTRODUCTION}

Parturition is a natural process as well as a challenging and high-risk event for both the cow and her offspring. As dairy cattle are not rigorously selected for calving ease, the prevalence of calving difficulties is higher in dairy cattle than in beef cattle (Garry, 2004), and dystocia rates are increasing all over the world (Mee, 2008). Besides several aspects of management, giving assistance at calving has a great importance. Early intervention has the potential to prevent stillbirths (Schuenemann et al., 2011), but unnecessary or premature intervention can also cause injuries in the birth canal due to the lack of proper soft tissue dilation (Mee, 2004). Other authors found that any assistance at calving is associated with an increased risk of stillbirth (Bicalho et al., 2007). Although the prevalence of dystocia may appear to be low internationally (between 4.1 and 13.7\%), calving assistance rates are high, varying between 10 and $>50 \%$ (both reviewed by Mee, 2008). Based on our personal experience, assisted calvings in Holstein-Friesian cows occur in around half of the births in Hungarian large-scale farms. It is thus questionable whether the assistance given is necessary in all of the cases and whether unnecessary assistance has consequences in terms of animal health.

Is seems that the timing of moving cows to the maternity pen before calving affects the duration of calving (Proudfoot et al., 2013) and the time periods from 
amniotic sac (AS) or feet appearance outside the vulva to birth are longer for assisted cows compared with unassisted ones (Schuenemann et al., 2011). However, only a few studies have examined the effects of any assistance provided at calving on the outcomes and the progress of delivery. In the present work, we were interested in finding whether the timing of obstetrical assistance has an effect on calving ease, the duration of the delivery process, and the health of the dam in the early postpartum period. We presumed that premature assistance at calving could affect the duration of the delivery process, the health status of the dam, and the vitality of the offspring. The possible well-being-related benefits of group calving over individual calving were also studied.

\section{MATERIALS AND METHODS}

\section{Animals and Housing}

The experiment was carried out in a commercial dairy farm in Hungary with around 1,000 lactating Holstein cows. One hundred seventy-six clinically healthy multiparous cows [means $\pm \mathrm{SD}$ (range); age $=5.8 \pm 0.7$ (4.9-6.8); parity $=3.2 \pm 0.3(2-4) ; \mathrm{BCS}=3.3 \pm 0.1$ (3-3.4)] that calved between September 10 and December 13, 2013, were allocated for the investigation. From $28 \mathrm{~d}$ before calving, preparturient cows were housed with preparturient heifers in a precalving group pen (measuring $45 \times 25 \mathrm{~m}$ ), which included 50 to 60 animals and was bedded with deep straw. Before calving, cows were fed a prepartum TMR ad libitum containing a dietary forage-to-concentrate ratio of $78: 22$ on a DM basis. After calving, cows were fed a postpartum TMR ad libitum with a 60:40 forage-to-concentrate ratio on a DM basis. Water was available ad libitum. During the first 5 DIM, cows were milked twice daily at 0400 and $1400 \mathrm{~h}$ in a 4-stall herringbone milking parlor operated with DeLaval Control Valve bucket milking machines (DeLaval International AB, Tumba, Sweden).

\section{Calving Management and Experimental Groups}

According to the farm management, cows calved in the group pen or, if assistance was provided, in a separated individual pen. Supervision of the dams during calving and the decision to move them into the maternity pen or to provide assistance was made by the farm personnel. Calving personnel moved cows to the separated maternity pen if the calving would have been disturbed by group mates or if assistance was required. The timing of this movement relative to calving was dependent on the course of calving (i.e., when signs of the imminent calving were visible; the onset of calving restlessness or rhythmic abdominal contractions and the release of the AS). Ten minutes after moving cows in the maternity pen, animals were examined to check presentation of the calf. When a malpresentation was evident (e.g., appearance of one foot outside the vulva), obstetrical assistance was performed by calving personnel immediately after AS appearance. Further observation of cows after examination was performed once every $30 \mathrm{~min}$. Calvings were divided into 4 groups based on the location of calving and the prevalence and timing of obstetrical assistance as follows:

(1) unassisted calving in an individual (maternity) pen (UCIP, $\mathrm{n}=42$ );

(2) unassisted calving in a group pen (UCG, $\mathrm{n}=$ 48);

(3) assisted calving in an individual pen with appropriately timed assistance provided without calving progress $70 \mathrm{~min}$ after the appearance of the AS or $65 \mathrm{~min}$ after the appearance of fetal hooves in the vulva (ACAP, $\mathrm{n}=50$ ); and

(4) assisted calving in an individual pen with inappropriately timed (premature) obstetrical assistance (earlier than in ACAP cows; ACIN, $\mathrm{n}=$ $36)$.

The ACAP and ACIN groups were defined taking into account the recommendations of Schuenemann et al. (2011) on the timing of obstetrical assistance. In our study, the start of obstetrical assistance was considered the time when at least 1 person assisted the cow in the calving pen using a calving rope or a calf puller, without leaving the pen for more than 2 min. Cows were similar in age, parity, and gestation length for all groups. In ACIN cows, the onset of obstetrical assistance ranged between 22 and $64 \mathrm{~min}(34.6 \pm$ $10.5)$ and between 5 and $48 \min (28.4 \pm 13.5)$ after AS and hooves appearance, respectively. In cases of ACIN calvings, staff started to assist mostly without notable behavioral signs of pain or distress of the cow. In ACAP cows, the onset of obstetrical assistance ranged between 76 and $89 \min (81.0 \pm 4.4)$ and between 68 and 82 min $(73.1 \pm 5.2)$ after AS and fetal hooves appearance, respectively. In this field study, the observation of AS or feet appearance outside the vulva were considered as clear and concrete landmarks that were easily identified. Cows in UCG groups calved without any human involvement at any point during the delivery process. Once born, calves were removed from their mothers within 30 min. Following calf removal, the dams were kept in postpartum pens for $5 \mathrm{~d}$ before being introduced to the milking herd. 


\section{Recorded Parameters of Calving}

Dystocia was defined as calving difficulty resulting from prolonged spontaneous calving or prolonged or severe assisted extraction (Mee, 2004). According to Mee (2008), assisted calving may be defined as a calving where assistance is rendered, although this may not result in dystocia. Following the above definitions, in ACAP and ACIN groups calvings with slight assistance right before delivery (where assistance was brief, traction slight, and the cow may otherwise have calved unassisted), and calvings when a prior examination was followed by a rapid (less than 2 min) and slight assistance to correct malpresentation were considered as normal calvings. Such slight assistance was provided for ACAP cows in 2 cases to correct malpresentation and the onset of this assistance was not considered when animals were divided into calving groups (see definition of calving assistance earlier). The incidence and degree of dystocia were recorded. (1) Eutocia (normal calving) was recorded after a combination of no assistance and slight assistance by 1 person (Mee et al., 2011). (2) Light dystocia was considered prolonged spontaneous calving ( $>2 \mathrm{~h}$ from hooves appearance to delivery) or calving assisted by 1 person without the use of mechanical traction, with moderate force. (3) Mild dystocia required assistance by 2 people without mechanical traction, with considerable force. (4) Severe dystocia was recorded after assistance by 3 people with considerable force or assistance with the use of mechanical extraction during delivery. During the study period, Caesarean section or fetotomy was not performed.

Duration of the stages of calving was recorded as follows: (1) time from the onset of calving restlessness to AS appearance, (2) time from AS appearance to birth, (3) time from hooves appearance to birth, and (4) the total duration of the calving process (between the onset of calving restlessness and delivery). The onset of calving restlessness was registered based on video recordings [2 day/night outdoor network bullet cameras installed above the group pen (Vivotek IP8331, Vivotek Inc., Taipei, Taiwan)], and established according to generally accepted behavioral predictors (Miedema et al., 2011).

The mean BCS of the dam was scored using the 5-point scoring system (Hady et al., 1994) following calving. The BW of the calf, as well as the incidence of stillbirth was also recorded immediately after birth. Calves that were born alive but died before $24 \mathrm{~h}$ of age were recorded as stillborn. Neonatal vitality was evaluated immediately after delivery and $24 \mathrm{~h}$ after birth using the vitality score as recommended by Szenci (1982). In this study only 3 categories were used: a score of 2 indicated normal tonicity, head erect, normal reflectory movements; a score of 1 indicated low tonicity, sternal recumbency with head requiring support, reduced number, and intensity of reflectory movements; and a score of 0 indicated toneless, head dropping, limbs extended, and cardiac activity absent (dead). Twenty-four hours after calving, the cows were examined clinically and the occurrence of retained placenta was recorded. Retained placenta was characterized by failure to release the placenta within $24 \mathrm{~h}$ of parturition. The occurrence of vulvovaginal laceration was also recorded at $48 \mathrm{~h}$ after calving [no laceration (1), and with laceration at the dorsal commissure or internal wall of the vulva or vagina $(2)]$.

\section{Statistics}

Statistical analysis was performed in the R 3.0.2 statistical environment and language (R Core Team, 2013). Data were tested for equality of error variances with the modified robust Brown-Forsythe Levene-type test based on the absolute deviations from the median. The Pearson Chi-squared test was used to evaluate the association between the proportion of dichotomous (overall dystocia rate, degrees of dystocia), binary (stillbirth ratio, retained placenta, vulvovaginal laceration), and categorical variables (BCS of the dam, vitality score of the calf immediately and $24 \mathrm{~h}$ after birth). Significant results were followed by multiple Z-tests to compare corresponding proportions and means of score data across calving groups. $P$-values for all pairwise comparisons were adjusted using the Bonferroni correction. The level of significance was set at 0.05. After verifying normality (Kolmogorov-Smirnov test), continuous variables (i.e., BW of the calf and the durations of the stages of the delivery process) were determined using ANOVA and pairwise comparison of means between calving groups was carried out with Tukey's post hoc test $(P<0.05)$.

\section{RESULTS AND DISCUSSION}

No twin calvings occurred during the experiment. The overall incidence of calving assistance was $48.8 \%$. The characteristics and outcomes of calvings, the BCS of the dam, and BW of the calves are presented in Table 1. Live BW of the calf and BCS of the dam immediately after birth did not differ across groups. Time from the onset of calving restlessness to AS appearance did not differ across groups. The time from AS and hooves appearance to birth as well as the total duration of the delivery process were shorter for UCG cows than for UCIP animals $(P=0.024, P=0.017$, and $P$ $=0.020$, respectively); this suggested that calving in a 
Table 1. The progress and the outcomes of calvings used in the study ${ }^{1}$

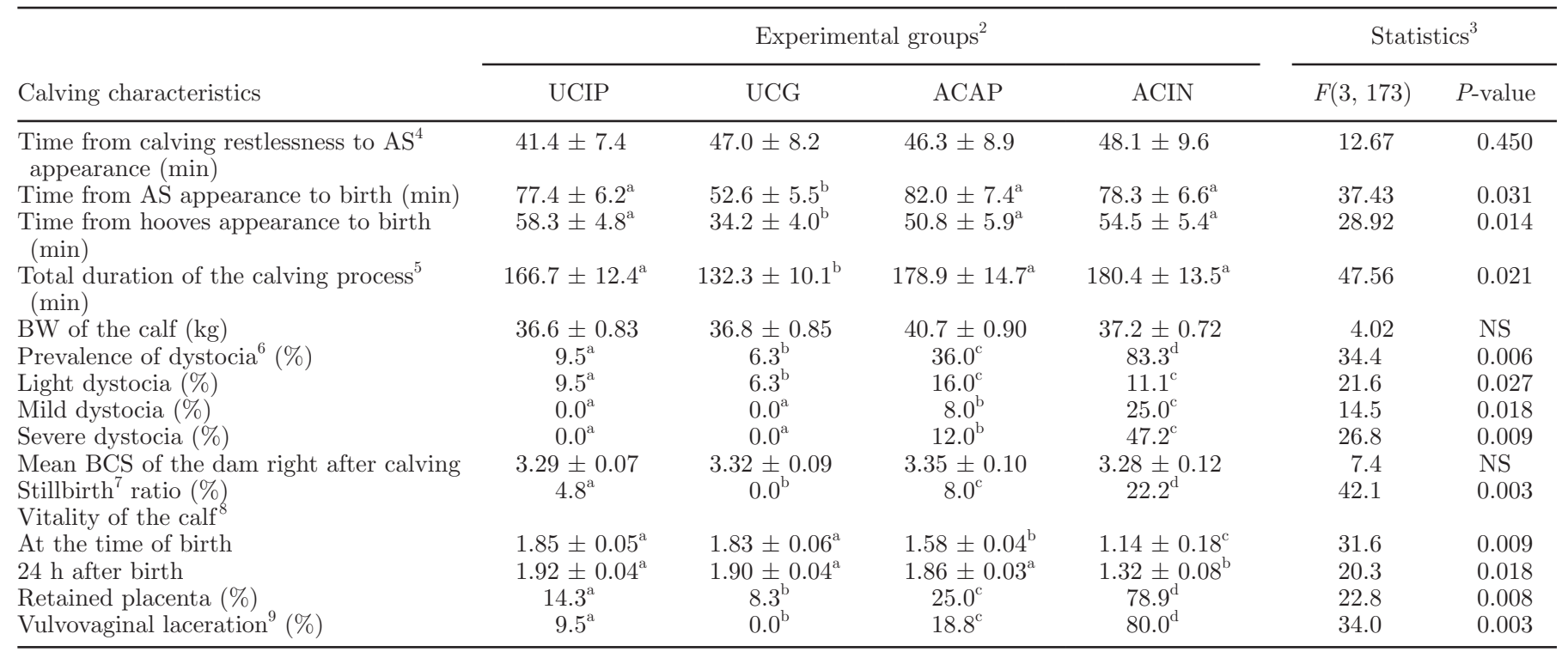

${ }^{\mathrm{a}-\mathrm{d}}$ Means with different superscripts within a row are significantly different $(P<0.05)$.

${ }^{1}$ Descriptive statistics are based on means $( \pm$ SEM) of nontransformed data.

${ }^{2} \mathrm{UCIP}=$ unassisted calving in an individual pen $(\mathrm{n}=42), \mathrm{UCG}=$ unassisted calving in a group pen $(\mathrm{n}=48)$, ACAP = assisted calving with appropriately timed obstetrical assistance $(\mathrm{n}=50)$, ACIN $=$ assisted calving with inappropriately timed obstetrical assistance $(\mathrm{n}=36)$.

${ }^{3} F$-statistics are based on results from the ANOVA. Statistical significances between groups are based on Tukey's test. $\chi^{2}$-statistics are based on the results of the Pearson Chi-squared test. Statistical significances between groups are based on the Z-tests.

${ }^{4} \mathrm{AS}=$ amniotic sac.

${ }^{5}$ From the onset of calving restlessness to birth.

${ }^{6}$ Light dystocia $=$ assistance by 1 person without the use of mechanical traction; mild dystocia = assistance by maximum of 2 persons; severe dystocia $=$ mechanical extraction or assistance by 3 persons.

${ }^{7}$ Stillbirth was defined as a calf born dead or dying within $24 \mathrm{~h}$ after birth.

${ }^{8}$ Newborn calf vitality was evaluated using a vitality score system on a scale from 0 to 2.

${ }^{9}$ Occurrence of vulvovaginal laceration was recorded at $48 \mathrm{~h}$ after calving: no laceration (1) or laceration at the dorsal commissure or internal wall of the vulva or vagina (2).

group might have benefits over calving in an individual pen in terms of the duration of the delivery process (Table 1). When focusing on the total duration of the calving, the differences were more pronounced between $\operatorname{ACAP}(P=0.008)$ and ACIN $(P=0.005)$ versus UCG cows.

The overall incidence of dystocia was $31.3 \%$ in the calvings studied. In 64.0 and $16.7 \%$ of ACAP and ACIN calvings, respectively, obstetrical intervention was provided to correct malpresentation of the calf or only slight assistance was provided; therefore, these events were not regarded as calving difficulties. The prevalence of dystocia was the lowest in UCG group, followed by UCIP dams, both below $10 \%$ (Table 1). In both unassisted groups, light dystocia events occurred (over $2 \mathrm{~h}$ between hooves appearance and delivery), whereas mild or severe difficulties were registered in cases of calvings requiring assistance. The proportion of dystocia was more than twice as high in ACIN cows than in ACAP animals $(P=0.004)$, and the proportion of severe dystocia was more than 4 times as high in ACIN cows than in cows that received appropriately timed assistance at calving $(P<0.001)$. Associations between parity and calving difficulties have been reported by several authors (Meyer et al., 2000; Pryce et al., 2006; Lombard et al., 2007); however, in our study, animals were balanced for parity between calving groups and only multiparous cows were used. In multiparous cows, dystocia is often related to twins or malpresentation of the calf (Mee, 2008); however, no twin calvings occurred during the experiment and, in cases of malpresentations ( $\mathrm{n}=2$, for ACAP cows), dystocia did not occur. The reason for this finding could be that calving personnel started to assist mostly without remarkable signs of pain of ACIN cows. In these cases, premature assistance might have prevented the proper dilation of the birth canal and disturbed the progress of normal delivery. The stillbirth ratio was higher in calves born to assisted dams than in those from spontaneous births (Table 1). In agreement with our findings, 
Schuenemann et al. (2011) observed increased incidence of stillbirths in assisted dams compared with cows with unassisted calvings. In ACIN cows, the proportion of stillbirths was higher than in ACAP cows $(P=0.007)$. The absence of stillbirth in UCG cows $(P<0.001$ compared with any other group) highlights the positive effect of group calving on calf survival.

No clinically evident signs of distress have been detected for calves that underwent spontaneous births or calves born from ACAP dams. All 140 calves from UCIP, UCG, and ACAP births were viable at birth because their mean vitality scores were consistent with the normal ranges reported for newborn calves (Szenci, 1982). Although ACAP calves had lower vitality scores than UCG and UCIP calves at birth $(P=0.023$ for both comparisons), a delayed recovery of vitality was evident in ACAP calves, with satisfactory vitality scores (>1.8) after $24 \mathrm{~h}$ with similar values as calves that underwent unassisted births (Table 1). Calves born from ACIN dams had lower vitality scores than calves born from UCG, UCIP, or ACAP dams at both birth $(P=$ $0.026, P=0.007$, and $P=0.006$, respectively) and 24 h thereafter $(P=0.014, P=0.010$, and $P=0.010$, respectively). In accordance with this finding, premature assistance was reported to be a risk factor for fetal trauma and stress (Schuijt, 1990), whereas others found that calves from assisted births are less vigorous than those delivered naturally (Barrier et al., 2012; Vannucchi et al., 2015).

The associations between the timing of obstetrical assistance (or any assistance given at calving) and postpartum health are not well studied. The increased risk of retained placenta has only been shown for dystocia (Lombard et al., 2003; Opsomer and de Kruif, 2009). We found a higher prevalence both of retained placenta and vulvovaginal laceration of the birth canal in assisted dams [i.e., ACAP $(P=0.016$ and $P=0.0 .12$, respectively) and ACIN animals $(P<0.001$ for both health disorders)] compared with UCIP cows. Both postpartum health problems had extreme high prevalence in ACIN cows (Table 1), suggesting that premature assistance has the potential to cause superficial injuries in the birth canal (Mee, 2004; Schuenemann et al., 2011). It is also important to note that, in UCG cows, no injuries occurred in the vulva or vagina and only 4 cases of retained placenta $(8.3 \%)$ were noted, proportions lower than in cows with unassisted calving in the maternity pen $(P<0.001$ and $P=0.028$, respectively).

Our results demonstrate that calving with appropriately timed assistance causes no serious impairment in calving ease, postpartum health of the dam, and in the vitality of the offspring. Although it needs further con- firmation, it seems that calving in a group has positive effects on postpartum health of the dam and survival of the offspring. Premature obstetrical assistance results in high prevalence of severe dystocia and has a negative effect on postpartum health of the dam and newborn calf vitality, emphasizing that appropriate time for assistance is paramount for peripartal well-being of both the dam and her offspring.

\section{ACKNOWLEDGMENTS}

The authors thank Ferenc Bodó, the owner of the farm for supporting the study, and the farm staff of Prograg Agrárcentrum Ltd. at Ráckeresztúr, Lászlópuszta, Hungary, for taking care of the animals during the experimental period. Levente Kovács was partly supported by the Research Center of Excellence-11476-3/2016/ FEKUT project (Budapest, Hungary).

\section{REFERENCES}

Barrier, A. C., E. Ruelle, M. J. Haskell, and C. M. Dwyer. 2012. Effect of a difficult calving on the vigour of the calf, the onset of maternal behaviour, and some behavioural indicators of pain in the dam. Prev. Vet. Med. 103:248-256.

Bicalho, R. C., K. N. Galvao, S. H. Cheong, R. O. Gilbert, L. D. Warnick, and C. L. Guard. 2007. Effect of stillbirths on dam survival and reproduction performance in Holstein dairy cows. J. Dairy Sci. 90:2797-2803.

Garry, F. B. 2004. An overview of animal welfare in the U.S. dairy industry. Pages 207-240 in The Well-Being of Farm Animals: Challenges and Solutions. G. J. Benson and B. E. Rollin, ed. Iowa State University Press, Ames.

Hady, P. J., J. J. Domecq, and J. B. Kaneene. 1994. Frequency and precision of body condition scoring in dairy cattle. J. Dairy Sci. $77: 1543-1547$.

Lombard, J. E., F. B. Garry, S. M. Tomlinson, and L. P. Garber. 2003. Relationship of dystocia to dairy cow health and productivity. J. Dairy Sci. 86(Suppl. 1):32. (Abstr.)

Lombard, J. E., F. B. Garry, S. M. Tomlinson, and L. P. Garber. 2007. Impacts of dystocia on health and survival of dairy calves. J. Dairy Sci. 90:1751-1760.

Mee, J. F. 2004. Managing the dairy cow at calving time. Vet. Clin. North Am. Food Anim. Pract. 20:521-546.

Mee, J. F. 2008. Prevalence and risk factors for dystocia in dairy cattle: A review. Vet. J. 176:93-101.

Mee, J. F., D. P. Berry, and A. R. Cromie. 2011. Risk factors for calving assistance and dystocia in pasture-based Holstein-Friesian heifers and cows in Ireland. Vet. J. 187:189-194.

Meyer, C. L., P. J. Berger, and K. J. Koehler. 2000. Interactions among factors affecting stillbirths in Holstein cattle in the United States. J. Dairy Sci. 83:2657-2663.

Miedema, H. M., M. S. Cockram, C. M. Dwyer, and A. I. Macrae. 2011. Behavioural predictors of the start of normal and dystocic calving in dairy cows and heifers. Appl. Anim. Behav. Sci. 131:14-19.

Opsomer, G., and A. de Kruif. 2009. Metritis and endometritis in high yielding dairy cows. Vlaams Diergeneeskd. Tijdschr. 78:83-88.

Proudfoot, K. L., M. B. Jensen, P. M. Heegaard, and M. A. G. von Keyserlingk. 2013. Effect of moving dairy cows at different stages of labor depending on behavior during parturition. J. Dairy Sci. 96:1638-1646. 
Pryce, J. E., B. L. Harris, S. Sim, and A. W. McPherson. 2006. Genetics of stillbirth in dairy calves. Proc. N.Z. Soc. Anim. Prod. 66:98-102.

R Core Team. 2013. R: A language and environment for statistical computing. R Foundation for Statistical Computing, Vienna, Austria. Accessed Feb. 3, 2015. http://www.R-project.org/

Schuenemann, G. M., I. Nieto, S. Bas, K. N. Galvão, and J. Workman. 2011. Assessment of calving progress and reference times for obstetric intervention during dystocia in Holstein dairy cows. J. Dairy Sci. 94:5494-5501.

Schuijt, G. 1990. Iatrogenic fractures of ribs and vertebrae during delivery in perinatally dying calves: 235 cases (1978-1988). J. Am. Vet. Med. Assoc. 197:1196-1202.
Szenci, O. 1982. Correlations between muscle tone and acid-base balance in newborn calves: experimental substantiation of a simple new score system proposed for neonatal status diagnosis. Acta Vet. Acad. Sci. Hung. 30:79-84.

Vannucchi, C. I., J. A. Rodrigues, L. C. G. Silva, C. F. Lúcio, and G. A. L. Veiga. 2015. Effect of dystocia and treatment with oxytocin on neonatal calf vitality and acid-base, electrolyte and haematological status. Vet. J. 203:228-232. 